V.V. Kniaziev, I.O. Postilnyk

National Technical University “Kharkiv Polytechnic Institute”, Kharkiv, Ukraine

\title{
THE CALIBRATION PROCEDURE FOR THE INDICATOR OF THE ELECTRIC FIELD FOR A THUNDERSTORM WARNING SYSTEM
}

The article presents a new version $X-1$ of the indicator of the electric field. The indicator is designed for use in the Thunderstorm Warning Systems. The principle of operation of the indicator X-1 is based on the corona discharge effect from metal rods. Calibration of the base variant of the device is carried out in high-voltage laboratory at use $H V$ stand BBC-1.2. Depending on the installation space required to carry out the calibration of the indicator. Calibration is carried out by calculation. To validate the results of calculations using the classical field strength meter, based on a rotary principle.

Key Words: thunderstorm warning system, field strength meter, effective alarm, lightning flash, warning level.

\section{INTRODUCTION}

As estimated that 24,000 people are killed by lightning strikes around the world each year and about 240,000 are injured [1]. As estimated 25 billion flashes of lightning strike is occurred every year across the U.S. On average, 49 people are killed by lightning in the U.S. each year. On average, 30 people are killed by lightning in the Ukraine each year. Lightning is the main killer among natural disasters in India. According to the government, at least 2,582 people died from lightning strikes, in 2014. In June 2016 Lightning strikes killed at least 120 people in four Indian states [2].

What can we do for reducing the number of victims? To create and make extensive use of Thunderstorm Warning Systems.

The need to create Thunderstorm Warning System very well grounded in the standard [3]: Natural atmospheric electric activity and in particular cloud-to-ground lightning poses a serious threat to living beings and property. Every year severe injuries and even deaths of humans are caused as a direct or indirect result of lightning. So, Lightning:

- may affect sport, cultural and political events attracting large concentrations of people. Events may have to be suspended and people evacuated in the case of a risk of thunderstorm;

- may affect industrial activities due to power outages and unplanned interruptions of production processes;

- may interrupt all kinds of traffic (people, energy, information, etc.);

- has led to a steady increase in the number of accidents per year due to the wider use of electric components that are sensitive to the effects of lightning (in industry, transportation and communication). In order to reduce this number of accidents and important material losses, it may be necessary in some circumstances, to disconnect certain equipment from any incoming installations;

- may be a hazard for activities with an environmental risk, for example: handling of sensitive, inflammable, explosive or chemical products.

- may be a cause of fire.

Four distinct stages can be identified during the thunderstorm life time cycle regarding detectable phenomena [3]: the initial phase (phase 1), the growth phase (phase 2), the mature phase (phase 3 ) and the dissipation phase (phase 4). The initial phase (Cumulus stage) is a phase of cloud electrification by means of electric charge separation within the cloud. The charges are distributed in regions within the cloud and produce a measurable electrostatic field at ground level. It is considered the first detectable phenomenon before a thunderstorm. Electrostatic fields may produce potential dangers such as electrostatic discharges even in the case of no lightning activity. The growth phase is characterized by the occurrence of first lightning discharge: intracloud (IC) or cloud-ground (CG). The first IC flashes appear after certain development of the charge regions in the cloud. However, in some situations there is no clear time delay between the first IC flash and the first CG flash. IC flashes typically represent the majority of the total lightning activity generated by a thunderstorm. Significant variation in the IC/CG rate is observed for individual storms. Mature phase is characterized by the presence of both CG and IC flashes. Dissipation phase is characterized by the decaying of both IC and CG flash rates and the reduction of the electrostatic field to the fair weather level.

Thunderstorm detectors are classified in relation with the detectable thunderstorm phases depending on the detectable phenomena [3]. There are several ways to look at the means to detect thunderstorms in general and lightning strikes in particular. One way is to look at the phase of the thunderstorm for which a detector is meant in particular. Another way is to compare the frequency 
range of the electromagnetic radiation emitted by a lightning strike with the frequency range detectable by a sensor. A third way is to look at techniques that a sensor uses to detect a lightning strike and to calculate its position.

For the classification of thunderstorm or lighting strike detectors, the following classes are defined [3]:

- Class A: detectors of class A detect a thunderstorm over its entire lifecycle (phases 1 to 4 );

- Class B: detectors of class B detect IC and CG flashes (phases 2 to 4 );

- Class C: detectors of class C detect CG flashes only (phases 3 and 4);

- Class D: detectors of class D detect CG flashes (phase 3) and other electromagnetic sources with very limited efficiency.

The classes are not related to the efficiency of the system. At the present time, the most widely used of lightning location system based on the detectors of class B and C. Such systems are designed for a set of statistical data, based on which it is possible to determine the spatial distribution of the cloud-to-ground discharges density $\left(\mathrm{N}_{\mathrm{g}}\right) . \mathrm{N}_{\mathrm{g}}$ is the primary descriptor of lightning incidence, needed in essentially all lightning protection studies. Multiple-station lightning locating systems (LLS) or lightning detection systems (LDS) are by far the best available tool for mapping $\mathrm{N}_{\mathrm{g}}[4,5]$. Those systems, besides locations, also report peak currents inferred from measured electric or magnetic fields.

However, even in these countries there is no a wide practice of local systems of crime prevention of the danger in a particular thunderstorm secluded location. Therefore, the development of specialized Thunderstorm Warning System is a new priority direction.

At the present time, in Ukraine there is no any infrastructure, which provides registration of lightning parameters and allows for warning people about the atmospheric processes that are highly likely to lead to under-occurrence of lightning in a particular area. Obviously, the information on the meteorological service does not meet the quality requirements of the warning process.

\section{The Purpose of The Work}

Create a warning system about the degree of danger of a lightning between the cloud and the earth's surface in a particular place at a particular time. The system is based on monitoring of the electromagnetic fields in the atmosphere settings.

\section{Analysis of Recent Publications}

\section{Principles of lightning location}

Potentially, LLS (or LDS) can be used to achieve this goal. Let us consider the known variants of such systems, with the analysis of opportunities and con- straints. The frequency ranges that are used in lightning detection are [3]:

- VLF: very low frequencies $(3 \mathrm{kHz}-30 \mathrm{kHz})$;

- LF: low frequencies $(30 \mathrm{kHz}-300 \mathrm{kHz})$;

- VHF: very high frequencies $(30 \mathrm{MHz}-300$ $\mathrm{MHz})$.

All these phenomena to be measured result in different sensor and location techniques. Those techniques may be distinguished as follows:

- MDF: magnetic direction finder;

- TOA: time of arrival;

- RFI: interferometry;

- FSM: field strength meter;

- RF: radio frequency signal strength measurements.

This list is not exhaustive.

In recent years, developed systems based on the use of a large number of sensors [6-8]. Moreover, the sensors are used as simple as possible. Such systems make it easy to integrate them to all comers. The results of monitoring lightning activity depicted on the map of the region in variants, similar to those presented in Fig. 1. Obviously, such systems have a more convenient for individual use.

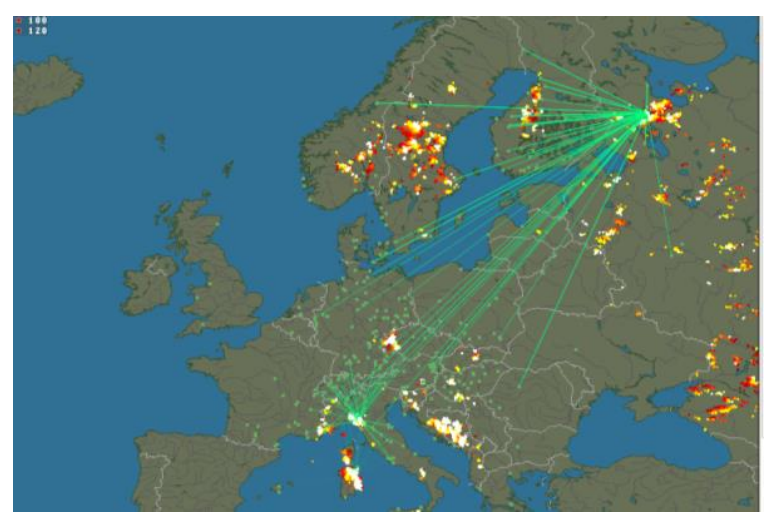

Fig. 1. Monitoring lightning activity from [7]

How to operate these systems? Consider the example of the WWLLN network [8]: System welcome offers of hosting a new WWLLN sensor to add to the list. All hosts receive all the worldwide data for their own research on monthly CDs. In return, each host provides the computer and meets any local expenses like power, Internet, and maintenance. However, do not think that a sensor on your own campus is going to give you lightning location data on its own. Only the whole network does that. Each lightning stroke location requires the time of group arrival (TOGA) from a least 5 WWLLN sensors. These sensors may be several thousand $\mathrm{km}$ distant from the stroke. The geographical arrangement of the sensors is important: a lightning stroke, which is enclosed by sensors, is much more accurately located than one that is not so enclosed. Clearly, a uniform spacing of sensors around the Earth is the ideal. Since the Earth is round, there are no edges: sensors surround every lightning stroke, but not necessarily 
by the sensors, which sense it. Typically, only about 15 to $30 \%$ of strokes detected by one sensor are detected by five or more. These strokes are usually the stronger ones. Recent research indicates detection efficiency for strokes about $30 \mathrm{kA}$ is approximately $30 \%$ globally. To cover the whole world by sensors spaced uniformly about $1000 \mathrm{~km}$ apart would require roughly 500 sensors. Presently system have 70 WWLLN sensors around the globe to detect sferic activity in the VLF band.

\section{Expansion possibilities LLS}

Analysis of the methods used, which are based on existing worldwide and national network of registration of lightning is presented in [5]. The results of the analysis clearly indicate that all the national networks have significant drawbacks in terms of our goal. Such systems require large financial investments and permanent staff it does not provide the main - rapid alert people in a particular place and particular time.

Currently, some firms proposed to use the electrostatic field sensors that react to achieve a certain level of field strength. Use the static and quasi-static electric fields range allows creating own network and integrating it into existing LLS networks.

Known sensor EFM-100 Atmospheric Electric Field Monitor produced by BOLTEK CORPORATION. Sensors (Fig 2) of this type have a fast rotating plate (rotational frequency reaches thousands of rotations per minute), which requires a large electric power supply, and creates significant problems in maintenance.

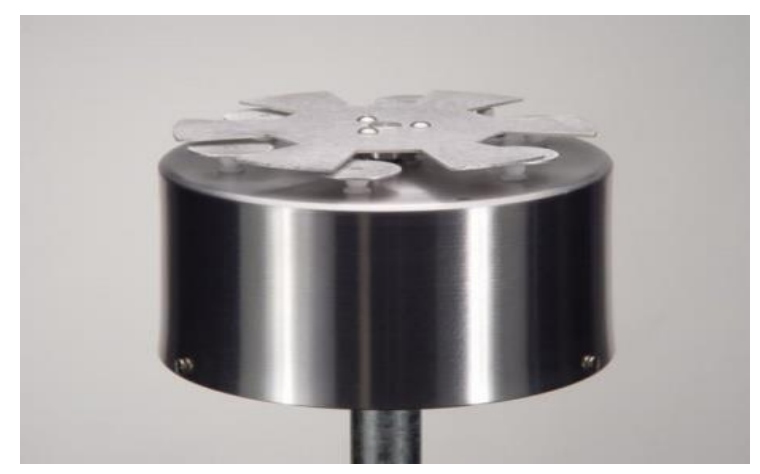

Fig. 2. EFM-100C Electric Field Mill Boltek (USA)

However, such devices are widely used for measuring of tension of the electrostatic fields. Therefore, we created an analogue and we use him for realization of calibration of the new rod detector.

The degree of reliability of the storm hazard assessment is determined by the correlation between the level of the electric field intensity and the speed of its growth. Therefore, one can assume that local systems available on the market do not provide the greatest possible degree of certainty about the storm warning of danger. A significant number of false positives can help ensure that people will not accept an alarm call as the truth, and simply will not respond to it. Especially, this subjective factor is important in places of recreation.

\section{THUNDERSTORM WARNING SYSTEM}

A thunderstorm warning system (TWS) is a system composed of thunderstorm detectors able to monitor the thunderstorm activity in the monitoring area and means of processing the acquired data to provide a valid alarm (warning) related to the lightning related events for a defined target area [3].

We propose to use the system, which is based on static and quasi-static electric field strength meters. The obvious advantage of such a system is that it monitors all four stages of thunderstorm development. It is important that this is the only option, which controls the first phase (the initial phase).

Typical thunderstorm cells are characterized by [3] diameters of $10 \mathrm{~km}$, cloud top altitudes of $12 \mathrm{~km}$ and a life cycle of less than $30 \mathrm{~min}$. But other types of thunderstorms such as multi-cell lines, cluster, super-cells, and mesoscale convective systems (MCS) have larger dimensions and durations that can reach several hours. Different types of thunderstorms tend to occur in different geographic regions.

Thunderstorm detectors are classified in relation to the thunderstorm phases depending on the detectable phenomena. However, a thunderstorm detector could detect one or several phenomena. We create a Class A detector. Class A detectors' purpose is to detect the first sign of a thunderstorm (Phase 1) useful for an early warning to take preventive actions, before the occurrence of any intra-cloud or cloud-to-ground lightning and during all the time when the risk of lightning exists. Detection is conducted by the measurement of the electrostatic field produced by the thunderstorm. The electrification of a thunderstorm or its presence itself produces an alteration of the fair weather electrostatic field. The atmospheric electrostatic field at ground level during fair-weather has a positive value of about $100 \mathrm{~V} \mathrm{~m}^{-1}$ to $150 \mathrm{~V} \mathrm{~m}^{-1}$ (atmospheric electricity sign convention) in a flat area, as the atmosphere above the Earth is positively charged. Under the electrified cloud of a thunderstorm, the electric field at ground level could reach several kilovolts per meter. Commonly the electrostatic field at ground level is screened from the field produced by the cloud due to the presence of screening layers and hence the electrostatic field usually remains below $10 \mathrm{~V} \mathrm{~m}^{-1}$.

A field strength meter (FSM), for detection purposes should have a minimum resolution of $200 \mathrm{~V} \mathrm{~m}^{-1}$ and should be able to measure an electric field of at least $\pm 20 \mathrm{kV} \mathrm{m}^{-1}$. Electrostatic field changes during the Initial Phase are relatively slow and sampling of the field every few seconds is sufficient. If for the application of the sensor information of the changes of the field is needed, a minimum sampling rate of one sample per 
second is recommended. The detector device should provide information about the electric field level. Some devices also could provide information about the field evolution in time.

The principle of operation of the indicator storm hazard, which is proposed by authors, based on the registration number of the corona current pulse from lightning rods. In [9], have determined dependence of the number of the corona current pulses on electric field strength, the length of the rod, the shape of its crosssectional area and shape of the top. Background information directly presented in digit of, which allows no more effort to measure the rate of change of the electric field strength. As a conversion element, using measuring shunt incorporated in the rod grounding circuit, or Rogowski coil covering the rod.

The photo of the electronic devise of the field strength meter (X-1) for Thunderstorm Warning System is presented in Fig. 3.

FSM X-1 has a number of significant advantages in comparison with analogues:

- The results obtained are practically independent of pressure, wind and rain;

- Inclusion of the device is carried out by an external source - corona current that does not require to keep the device in a fully powered on;

- Receiving device in the form of a metal rod is also the broadband receiving antenna atmospherics that allows tracking of storm fronts at distances up to $200 \mathrm{~km}$;

- Low cost component elements.

Typical temporary form of corona discharge from the air terminal at the time of thunderstorm is shown in Fig.9. Along the $\mathrm{X}$-axis is located the current time (Kiev), along the Y-axis - the level of the corona current pulses. Waveform consists of a large number of individual pulses, which are not visible on the scan presented. Timing a single pulse is $10 / 50 \mathrm{~ns}$. The pulse amplitude is in the range of $50-150 \mathrm{~mA}$.

The high level of lightning danger is achieved when the number of pulses of more than 1,000.

Creating a national thunderstorm warning system proposed to be carried on a "bottom-up", due to the installation of a large number of simple and cheap local elements, which are combined into a multi-level network via a data transmission via cellular networks and the internet. Only such a concept provides the possibility of establishing rapid alert systems.

Each local member provides early warning of storm danger in the place where it is installed, by supplying an audio and / or visual alarm, ensures the distribution of warning SMS-messages on a mobile device located in the area of cellular tower that serves element of the system, and transmits national information to the server system.

It presented a pilot version of the implementation of the local system based on electric field strength meter $(\mathrm{X}-1)$, based on counting the number of streamers from the rod air terminal. However, we consider other options of technical solutions.
The authors consider it advisable to unite the TWS system with the one of the new LLS systems [6-8]. This will significantly increase the reliability of alarm.

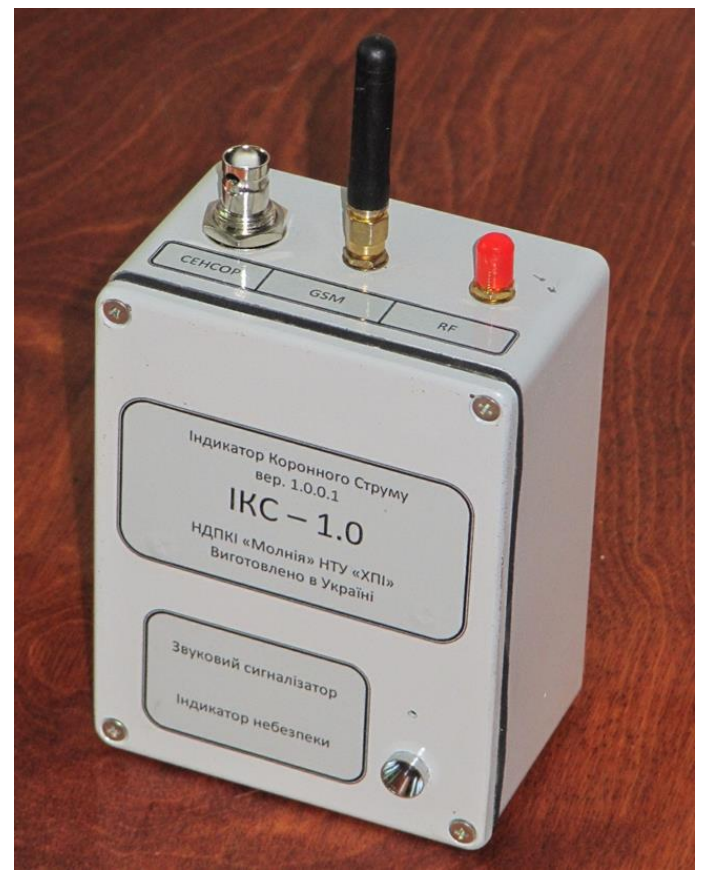

Fig. 3. The electronic devise of the field strength meter (X-1)

Calibration of the base variant of the device is carried out in high-voltage laboratory at use $\mathrm{HV}$ stand BBC-1.2. Depending on the installation space required to carry out the calibration of the indicator. Calibration is carried out by calculation. To validate the results of calculations using the classical field strength meter, based on a rotary principle (Fig.2).

The works carried out in the framework of the grant of the Ministry of Education and Science of Ukraine (\#0115U000611).

\section{REFERENCES}

[1]. Ronald L. Holle. Annual rates of lightning fatalities by country. 20th International Lightning Detection Conference. 21-23 April 2008. Tucson, Arizona, USA.

[2] www.edition.cnn.com/2016/06/22/asia/indialightning-deaths/

[3] Standard IEC 62793:2016 Protection against lightning Thunderstorm warning system.

[4] Amitabh Nag, Martin J. Murphy, Wolfgang Schulz, Kenneth L. Cummins, "Lightning locating systems: Characteristics and validation echniques", Proc. 2014 International Conference on Lightning Protection (ICLP), Shanghai, China, 2014. P. 824-836.

[5] Vladimir Rakov, "An Overview of the Performance Characteristics of Lightning Detection Systems", V Russian Conference on Lightning Protection // Invited presentation. St. Petersburg, Russia, May 16-19, 2016.

[6] www.lightningmaps.org

[7] http://ru.blitzortung.org

[8] http://wwlln.net/

[9] Chernukhin O.Yu. The Streamer Corona From The Rod Lightning Arresters / O.Yu. Chernukhin, V.V. Kniaziev // East European Scientific Journal, No.6, 2016. P.39-47. 